\title{
Optimal Multiplexing Gain of $K$-user Line-of-Sight Interference Channels with Polarization
}

\author{
Sung ho Chae, Sang Won Choi, and Sae-Young Chung \\ School of EECS, KAIST, 335 Gwahangno, Yuseong-gu, Daejeon, 305-701, South Korea \\ Email: shchae@kaist.ac.kr, ace1905@kaist.ac.kr, and sychung@ee.kaist.ac.kr
}

\begin{abstract}
We consider the multiplexing gain (MUXG) of the fully connected $K$-user line-of-sight (LOS) interference channels (ICs). A polarimetric antenna composed of 3 orthogonal electric dipoles and 3 orthogonal magnetic dipoles is considered where all 6 dipoles are co-located. In case of $K$-user IC with single polarization, the maximum achievable MUXG is $K$ regardless of the number of transmit and receive antennas because of the key-hole effect. With polarization, a trivial upper bound on the MUXG is $2 K$. We propose a zero forcing (ZF) scheme for the $K$-user LOS IC, where each user uses one or more polarimetric antennas. By using the proposed $\mathrm{ZF}$ scheme, we find minimal antenna configurations that achieve this bound for $K \leq 5$. For $K>5$, we show that the optimal MUXG of $2 K$ is achieved with $M=\left\lceil\frac{K+1}{6}\right\rceil$ polarimetric antennas at each user.
\end{abstract}

\section{INTRODUCTION}

In multi-user communications, resources such as time, frequency, and antennas need to be allocated to each user properly. One simple way is to allocate resources orthogonally. However, it can be very inefficient. To improve the performance further resources can be shared, which becomes more important as the number $(K)$ of users increases. Studying the interference channel (IC) can give us insights on how to manage interference better.

Exact capacity characterization of the Gaussian IC is unknown in general. However, approximate characterization by finding the optimal multiplexing gain has been found for many cases recently. For example, zero forcing has been shown to be efficient for $K$-user multiple-input multiple-output (MIMO) Gaussian IC [2]. Recently, interference alignment (IA) [3][6] when $K \geq 3$ has been shown to achieve the optimal degrees of freedom (DOF) of $\frac{K}{2}$ for time-varying channels. Furthermore, the IA scheme for line-of-sight (LOS) channels has been developed in [7].

MIMO is in general helpful for improving the multiplexing gain. However, in LOS channels, e.g., roof-top antennas, it is more challenging to achieve a higher multiplexing gain since regardless of the number of antennas the multiplexing gain can only be one in a point-to-point setup due to the key hole effect. Polarization can increase the DOF in this situation. It can provide two-fold increase in the DOF in a LOS environment. In [8], the authors showed that up to 6 DOF can be obtained by using a single polarimetric antenna in a scattering environment. In [9], the multiplicative gain in DOF was studied for different array geometry when polarimetric antennas are used. The results showed that the multiplicative

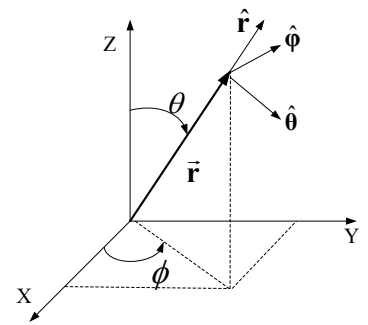

Fig. 1. Spherical coordinate system and corresponding unit vectors

gain from polarization depends on the array geometry and the scattering condition.

In this paper, we focus on the MUXG of the fully connected $K$-user LOS IC. We propose a new interference cancellation scheme using the polarimetric antenna studied in [8], [9]. Due to the key hole effect, the total MUXG in the $K$-user LOS IC is upper bounded by $K$ with single polarization. If we use polarimetric antennas at each user, we observe that a trivial upper bound on the DOF is now $2 K$. In this paper, we find minimal antenna configurations to achieve this trivial upper bound. For $K \leq 5$, we show some antenna configurations to achieve the optimal MUXG of $2 K$. We also show that for $K>5$, we need $\left\lceil\frac{K+1}{6}\right\rceil$ polarimetric antennas per node to achieve the optimal MUXG.

\section{Channel Model}

We consider a polarimetric antenna composed of 3 orthogonal electric dipoles and 3 orthogonal magnetic dipoles that are all co-located as in [8], [9]. Note that since the space is 3 dimensional we can try to have up to 3 electric and 3 magnetic dipoles at the same location to construct a polarimetric antenna. We interpret this polarimetric antenna as a vector point source having 6 elements.

We will first see the radiated electric fields from each electric dipole and magnetic dipole. For convenience, a spherical coordinate is considered throughout this paper as in Fig. 1. Consider electric dipoles oriented along the $x-, y$ - and $z$ axis, which are positioned symmetrically at the origin. We assume all the electric dipoles are half-wave dipoles, and their maximum electric currents are $I_{e 1}, I_{e 2}$, and $I_{e 3}$, respectively. Then, the radiated electric fields from each electric dipole 
measured at a point $\vec{r}$ in the far-field are given by [10]

$$
\begin{aligned}
E_{1}=E_{e}^{x} & \simeq j \sqrt{\frac{\mu}{\epsilon}} \frac{I_{e 1} e^{-j k r}}{2 \pi r}[-\cos \theta \cos \phi \hat{\theta}+\sin \phi \hat{\phi}] \\
& =C_{e 1} \frac{e^{-j k r}}{r} \mathbf{a}_{1}(\theta, \phi), \\
E_{2}=E_{e}^{y} & \simeq j \sqrt{\frac{\mu}{\epsilon}} \frac{I_{e 2} e^{-j k r}}{2 \pi r}[-\cos \theta \sin \phi \hat{\theta}-\cos \phi \hat{\phi}] \\
& =C_{e 2} \frac{e^{-j k r}}{r} \mathbf{a}_{2}(\theta, \phi),
\end{aligned}
$$

and

$$
E_{3}=E_{e}^{z} \simeq j \sqrt{\frac{\mu}{\epsilon}} \frac{I_{e 3} e^{-j k r}}{2 \pi r}[\sin \theta \hat{\theta}]=C_{e 3} \frac{e^{-j k r}}{r} \mathbf{a}_{3}(\theta, \phi),
$$

respectively, where $k$ denotes the wave number, $\sqrt{\frac{\underline{\mu}}{\epsilon}} \approx 377 \Omega$ is the impedance of free space, $r=|\vec{r}|$ is the distance between the observation point and the origin, and $C_{e i}=j \sqrt{\frac{\mu}{\epsilon}} \frac{I_{e i}}{2 \pi}, \forall i=$ $1,2,3$.

Similarly, the radiated electric fields from magnetic dipoles with the maximum magnetic current $I_{m 4}, I_{m 5}$, and $I_{m 6}$ are given by

$$
\begin{aligned}
E_{4}=E_{m}^{x} & \simeq j \sqrt{\frac{\mu}{\epsilon}} \frac{I_{m 4} e^{-j k r}}{2 \pi r}[\sin \phi \hat{\theta}+\cos \theta \cos \phi \hat{\phi}] \\
& =C_{m 4} \frac{e^{-j k r}}{r} \mathbf{a}_{4}(\theta, \phi), \\
E_{5}=E_{m}^{y} & \simeq j \sqrt{\frac{\mu}{\epsilon}} \frac{I_{m 5} e^{-j k r}}{2 \pi r}[-\cos \phi \hat{\theta}+\cos \theta \sin \phi \hat{\phi}] \\
& =C_{m 5} \frac{e^{-j k r}}{r} \mathbf{a}_{5}(\theta, \phi),
\end{aligned}
$$

and

$E_{6}=E_{m}^{z} \simeq j \sqrt{\frac{\mu}{\epsilon}} \frac{I_{m 6} e^{-j k r}}{2 \pi r}[-\sin \theta \hat{\phi}]=C_{m 6} \frac{e^{-j k r}}{r} \mathbf{a}_{6}(\theta, \phi)$

from duality theorem, where $C_{m i}=j \sqrt{\frac{\mu}{\epsilon}} \frac{I_{m i}}{2 \pi}, \forall i=4,5,6$. However, since there are no known magnetic currents in nature, magnetic current is purely a mathematical notion used to explain the motion of magnetic charges creating magnetic current, when compared to their dual quantities of moving electric charges giving rise to electric current [11]. In fact, we can generate the equivalent radiation pattern by a closed loop of electric current. The equivalent relationship between the magnetic current $I_{m}$ and the electric current of the loop antenna $I_{l}$ is given by $I_{m} \lambda / 2=j \pi a(2 \pi f)^{2} \mu_{0} I_{l}$, where $a$ is the radius of the loop antenna, $f$ is the operating frequency, and $\mu_{0}$ is permeability of free space. Note that the maximum electric (magnetic) currents of each dipole are determined by their transmit powers.

We now consider the fully connected $K$-user LOS IC with polarimetric transmit and receive antennas. Transmitter $i$ tries to communicate with receiver $i$ that is getting interference from all the other transmitters, where $\forall i=1,2, \cdots, K$, and each transmitter and receiver uses $M$ polarimetric antennas. In addition, we assume all channel coefficients are fixed during the communication duration (time invariant) and known to all transmitters and receivers. Then, the input and output relationship is given by

$$
\mathbf{Y}^{[j]}=\sum_{i=1}^{K} \mathbf{H}^{[i j]} \mathbf{X}^{[i]}+\mathbf{N}^{[i]}
$$

where $\mathbf{X}^{[i]}$ is the $6 M \times 1$ signal vector at the transmitter $i$, $\mathbf{H}^{[i j]}$ is the $6 M \times 6 M$ polarization matrix from the transmitter $i$ to the receiver $j$, and $\mathbf{Y}^{[j]}$ is the $6 M \times 1$ the received signal vector at the receiver $j$. The noise vector $\mathbf{N}^{[j]}$ is the additive white Gaussian with zero mean and covariance of $\mathbf{I}_{6 M}$, where $\mathbf{I}_{N}$ denotes the identity matrix of size $N \times N$.

In this paper, we assume that all the transmitters and receivers are located in the azimuth plane $\left(\theta=\frac{\pi}{2}\right)$ only. Note that this assumption holds roughly for typical LOS channels found in cellular networks.

In order to see the characteristics of polarization matrix, fix $i$ and $j$, and set $M=1$. Note that the $6 \times 6$ polarization matrix $\mathbf{H}^{[i j]}$ shows the voltage response of each component of the receiver $j$ due to the incident wave radiated by each component of the transmitter $i$. Let $a^{[i j]}$ denote the attenuation along the LOS path between transmitter $i$ and receiver $j$, and let $\phi_{i j}$ denote the angle between the transmitter $i$ and the receiver $j$. Then, the channel matrix $\mathbf{H}^{[i j]}$ is given by

$$
\mathbf{H}^{[i j]}=a^{[i j]} e^{-j k r_{[i j]}}\left[a_{\hat{\theta}}^{T} a_{\hat{\theta}}+a_{\hat{\phi}}^{T} a_{\hat{\phi}}\right]
$$

where $r_{[i j]}$ denotes the distance between the transmitter $i$ and the receiver $j$, and the operator $(\cdot)^{T}$ denotes the transpose of a matrix. In addition, we have $\left[\begin{array}{llll}\mathbf{a}_{1}\left(\frac{\pi}{2}, \phi_{i j}\right) & \mathbf{a}_{2}\left(\frac{\pi}{2}, \phi_{i j}\right) & \cdots & \mathbf{a}_{6}\left(\frac{\pi}{2}, \phi_{i j}\right)\end{array}\right]=a_{\hat{\theta}} \hat{\theta}+a_{\hat{\phi}} \hat{\phi}$ where $a_{\hat{\theta}}=\left[\begin{array}{llllll}\sin \left(\phi_{i j}\right) & -\cos \left(\phi_{i j}\right) & 0 & 0 & 0 & -1\end{array}\right]$ and $a_{\hat{\phi_{i j}}}=\left[\begin{array}{llllll}0 & 0 & 1 & \sin \left(\phi_{i j}\right) & -\cos \left(\phi_{i j}\right) & 0\end{array}\right]$. Note that $a_{\hat{\theta}}$ and $a_{\hat{\phi}}$ represent the relative magnitude of electric fields polarized in $\hat{\theta}$ and $\hat{\phi}$ directions, respectively, which are radiated by each component of the transmit polarimetric antenna with the same amount of current. We see that electric dipoles oriented along $x$ - and $y$-axis and magnetic dipole oriented along $z$ axis transmit horizontally polarized electric fields while the others transmit vertically polarized electric fields. Clearly, the rank of this matrix is 2 even though we use 6 different dipole components since there are only two degrees of freedom for a fixed propagation direction. Moreover, the channel matrix is symmetric due to the reciprocity of transmitter and receiver.

We follow the conventional definition of achievable rate and capacity region, which is omitted in this paper. The multiplexing gain $\Gamma[1]$ of the $K$-user LOS IC is defined as

$$
\Gamma=\lim _{\mathrm{SNR} \rightarrow \infty} \frac{R_{+}(\mathrm{SNR})}{\log (\mathrm{SNR})},
$$

where $R_{+}(\mathrm{SNR})$ is a sum rate at signal-to-noise ratio (SNR), where SNR is defined as the ratio of the total power across all transmitters and the noise variance at each receiver.

In this paper, we focus on the optimal MUXG for $K$ user LOS interference channel using polarimetric antennas. Our goal is to find the minimum antenna configurations that 


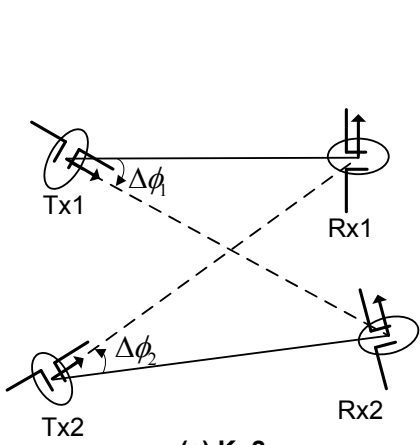

(a) $\mathrm{K}=2$

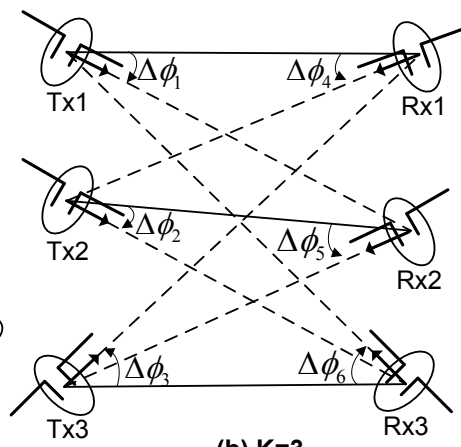

(b) $\mathrm{K}=3$

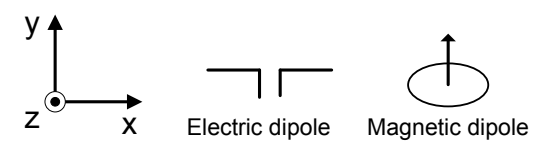

Fig. 2. Optimal antenna placement for $\mathrm{K}=2$ and $\mathrm{K}=3$ cases. Each transmitter and receiver is aligned along the cross-link direction which each of them wants to remove.

achieve the optimal MUXG of $2 K$. Note that without polarimetric antennas we can only achieve the MUXG of $K$ instead of $2 K$ in a LOS environment even if we allow to use multiple polarimetric antennas at each node. We propose two different scenarios, optimal antenna placement and fixed antenna placement. We will show the minimum antenna configuration under these scenarios, respectively. Optimal antenna placement means optimally adjusting the orientation of each dipole component physically. Such manual adjustment of dipole direction may be feasible if node locations are exactly known and do not change. In our second scenario, fixed antenna placement, all antenna directions are fixed independent of node locations. In this scenario, we may need more dipoles than in optimal antenna placement scenario.

\section{Optimal Antenna Placement}

In this section, we find minimal antenna configurations need to achieve the trivial upper bound of $2 K$ on the DOF in the optimal antenna placement scenario. Note that we need at least 2 dipoles per user to achieve the goal. Consider the example of 2 -user $(K=2)$ case as shown in Fig. 2-2.(a). Assume that all the transmitters and receivers have one electric and one magnetic dipoles that are co-located such that they are oriented along the direction of its direct link initially. In this case, the electric dipole is used to generate vertically polarized electric field, while the magnetic dipole generates horizontally polarized electric field. The key idea is based on the fact that the radiated electric field is always perpendicular to the direction of propagation, and the its magnitude is proportional to the projection of the antenna orientation to the field direction. Therefore, if the dipoles of transmitters 1 and 2 are physically rotated by $\Delta \phi_{1}\left(=\phi_{12}-\phi_{11}\right)$ and $\Delta \phi_{2}\left(=\phi_{21}-\phi_{22}\right)$, respectively where $\phi_{i j}$ denotes the angle between transmitter $i$ and receiver $j$, the transmitters do not radiate interfering signal to nondesired receivers. Since the interference is already canceled by each transmitter, the dipoles of receivers are rotated by
90 degrees to get the maximum antenna gain. It is clear that the role of the transmitters and the receivers can be swapped. Then, after adjusting the orientation of each dipole properly, we have the following channel matrices

$$
\mathbf{H}^{[11]}=a^{[11]} e^{-j k r_{[11]}}\left[\begin{array}{cc}
\sin \left(\Delta \phi_{1}\right) & 0 \\
0 & \sin \left(\Delta \phi_{1}\right)
\end{array}\right]
$$

and

$$
\mathbf{H}^{[22]}=a^{[22]} e^{-j k r_{[22]}}\left[\begin{array}{cc}
\sin \left(\Delta \phi_{2}\right) & 0 \\
0 & \sin \left(\Delta \phi_{2}\right)
\end{array}\right]
$$

while both $\mathbf{H}^{[12]}$ and $\mathbf{H}^{[21]}$ become null matrices. We see that if $\phi_{11} \neq \phi_{12}$ and $\phi_{22} \neq \phi_{21}, 2$ DOF per each user can be achieved. One can see that there can be other proper antenna configurations for achieving the same MUXG. Suppose the first user uses one electric and one magnetic dipole that are co-located and oriented along $z$ - and $x$-axis, respectively while the second user uses the same dipole set as the previous case. In this case, the channel matrix is given by

$$
\mathbf{H}^{[11]}=a^{[11]} e^{-j k r_{[11]}}\left[\begin{array}{cc}
1 & 0 \\
0 & \sin \left(\Delta \phi_{1}\right)
\end{array}\right]
$$

and

$$
\mathbf{H}^{[22]}=a^{[22]} e^{-j k r_{[22]}}\left[\begin{array}{cc}
\sin \left(\Delta \phi_{2}\right) & 0 \\
0 & \sin \left(\Delta \phi_{2}\right)
\end{array}\right]
$$

and the optimal MUXG is also achieved. However, if transmitter and receiver use electric dipoles only, we cannot remove the interference from the other user.

We can easily extend this scheme to $K=3$ by using same set of dipoles. However, since there are 2 interference links per each user in this case, the dipoles of both transmitters and receivers need to be rotated in order to remove all the interferences. One proper antenna configuration for $K=3$ is depicted in Fig. 2-(b), and the following channel matrices can be easily obtained for this case:

$$
\mathbf{H}^{[i i]}=a^{[i i]} e^{-j k r_{[i i]}}\left[\begin{array}{cc}
\lambda^{[i i]} & 0 \\
0 & \lambda^{[i i]}
\end{array}\right], \quad \forall i=1,2,3
$$

where $\lambda^{[11]}=\sin \left(\phi_{11}-\phi_{12}\right) \sin \left(\phi_{11}-\phi_{21}\right), \lambda^{[22]}=\sin \left(\phi_{22}-\right.$ $\left.\phi_{21}\right) \sin \left(\phi_{22}-\phi_{12}\right)$, and $\lambda^{[33]}=\sin \left(\phi_{33}-\phi_{31}\right) \sin \left(\phi_{33}-\phi_{13}\right)$ while the other channel matrices are null. Similar to $K=$ 2 case, we achieve the optimal MUXG of $2 K$ by rotating dipoles properly. Observe that unlike 2-user case, we cannot use electric or magnetic dipole oriented along $z$-axis since their radiation patterns are omni-directional in the azimuth plane. In summary, we have the following proposition for optimal placement.

Proposition 1: For $K \leq 3$ with optimal antenna placement, the following antenna configuration at each user achieves the optimal MUXG of $2 K$.

$$
\text { - }\left(e_{x} \text { or } e_{y}\right)+\left(m_{y} \text { or } m_{y}\right)
$$

where $e$ and $m$ denote electric and magnetic dipoles respectively, and the subscript denotes the orientation of each dipole.

When $K$ is greater than 3 , we can select a proper subset of 3 users to achieve up to 6 degrees of freedom. 


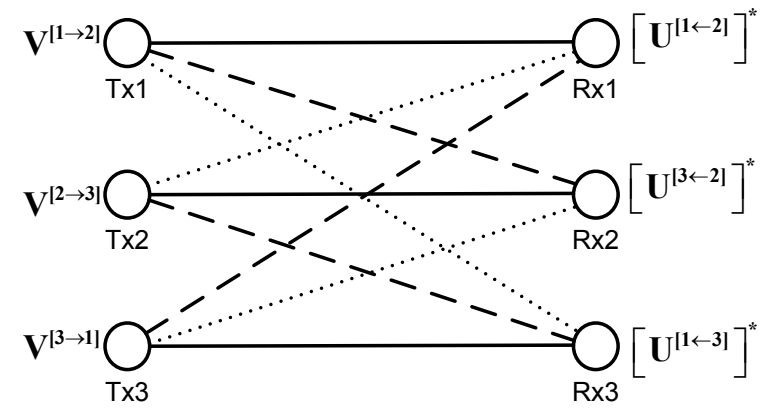

Fig. 3. Fixed antenna placement with single polarimetric antenna for $K=3$. The $\mathrm{ZF}$ beam-forming matrices for each transmitter and receiver are depicted. Dashed line (--) denotes the interference links nulled by receivers while dotted $(\cdots)$ line represents the interference links nulled by transmitters.

The proposed zero forcing scheme is simple and intuitive, but we have to adjust dipole orientation physically whenever the location of either a transmitter and receiver is changed. Therefore, this scheme can only be feasible if there is no mobility. Furthermore, we cannot use this scheme when $K>3$. In Section IV, we explain how to solve these problems by using more polarimetric antenna elements.

\section{Fixed Antenna Placement: $M=1$ Case}

It is easy to see that the electric (magnetic) dipoles oriented along $x$ - and $y$-axis together can create the electric field generated from an arbitrary rotated dipole in the azimuth plane. Thus, instead of rotating dipoles physically, we can obtain the same interference canceling effect by using more polarimetric antenna elements whose orientation is fixed. In this section, we focus on the case where $M=1$.

The optimal antenna placement scheme depicted in Fig. 2 . (b) can be replaced by simple ZF beam-forming scheme. The key idea is that both transmitter and receiver can eliminate a certain number of its interferences under our scheme. From now on, we will explain the proposed $\mathrm{ZF}$ beam-forming scheme for transmitter and receiver, respectively.

Consider the 3-user case first. One can easily see that 4 dipole components are at least required, 2 for vertical polarization and 2 for horizontal polarization. We assume each user activates 2 electric and 2 magnetic dipoles oriented along $x$ - and $y$-axis. Then, each effective channel becomes the $4 \times 4$ matrix whose rank is 2 . Assume the transmitter $i$ tries to null out the interference to the receiver $j$. Then, the channel matrix $\mathbf{H}^{[i j]}$ is given by

$$
\mathbf{H}^{[i j]}=a^{[i j]} e^{-j k r_{[i j]}}\left[\begin{array}{cc}
\mathbf{A}^{[i j]} & \mathbf{O}_{4 \times 4} \\
\mathbf{O}_{4 \times 4} & \mathbf{A}^{[i j]}
\end{array}\right]
$$

where

$$
\mathbf{A}^{[i j]}=\left[\begin{array}{cc}
\sin ^{2}\left(\phi_{i j}\right) & -\sin \left(\phi_{i j}\right) \cos \left(\phi_{i j}\right) \\
-\sin \left(\phi_{i j}\right) \cos \left(\phi_{i j}\right) & \cos ^{2}\left(\phi_{i j}\right)
\end{array}\right]
$$

is $2 \times 2$ matrix whose rank is 1 , and $\mathbf{O}_{4 \times 4}$ denotes the $4 \times 4$ zero matrix. We can easily find one proper $\mathrm{ZF}$ beam-forming matrix for the transmitter $i$ given by

$$
\mathbf{V}^{[i \rightarrow j]}=\left[\begin{array}{cc}
\cos \left(\phi_{i j}\right) & 0 \\
\sin \left(\phi_{i j}\right) & 0 \\
0 & \cos \left(\phi_{i j}\right) \\
0 & \sin \left(\phi_{i j}\right)
\end{array}\right]
$$

Similarly, since the channel is symmetric, if receiver $i$ intends to cancel the interference from the transmitter $k$ where $k \neq$ $i$ the beam-forming matrix for the receiver $i$ can be easily obtained as

$$
\mathbf{U}^{[k \leftarrow i]}=\left[\begin{array}{cc}
\cos \left(\phi_{k i}\right) & 0 \\
\sin \left(\phi_{k i}\right) & 0 \\
0 & \cos \left(\phi_{k i}\right) \\
0 & \sin \left(\phi_{k i}\right)
\end{array}\right] .
$$

Therefore, after applying the ZF beam-forming for user $i$, we have the equivalent parallel channel given by

$$
\begin{aligned}
\boldsymbol{\Lambda}^{[i i]} & =\left[\mathbf{U}^{[k \leftarrow i]}\right]^{*} \mathbf{H}^{[i i]} \mathbf{V}^{[i \rightarrow j]} \\
& =a^{[i i]} e^{-j k r_{[i i]}}\left[\begin{array}{cc}
\lambda^{[i i]} & 0 \\
0 & \lambda^{[i i]}
\end{array}\right]
\end{aligned}
$$

where $\lambda^{[i i]}=\sin \left(\phi_{i i}-\phi_{i j}\right) \sin \left(\phi_{i i}-\phi_{k i}\right)$, and the operator $(\cdot)^{*}$ denotes the complex conjugate transpose of a matrix. The detailed ZF beam-forming for each transmitter and receiver are summarized in Fig. 3. Note that $\boldsymbol{\Lambda}^{[i i]}$ has two non-zero diagonal elements unless $\sin \left(\phi_{i i}-\phi_{i j}\right)$ or $\sin \left(\phi_{i i}-\phi_{k i}\right)$ are zero $\forall i=1,2,3$. Furthermore, one can easily observe that this ZF beam-forming scheme achieves the exactly same DOF as in optimal antenna placement scenario.

In addition, it is obvious that the proposed $\mathrm{ZF}$ method can be also applied to $K=2$ case. For $K=2$ case, only transmitters or receivers need to perform $\mathrm{ZF}$ beam-forming because there is only one interference link per user.

It is worth to mention that we can also use 3 orthogonal electric dipoles plus 1 magnetic dipole oriented along $x$ - or $y$-axis, or 3 orthogonal magnetic dipoles plus 1 electric dipole oriented along $x$ - or $y$-axis instead to achieve the optimal MUXG of 6 since there are 2 dipole components for each vertical and horizontal polarization, respectively. However, if we use 3 orthogonal electric dipoles plus 1 magnetic dipole oriented along $z$-axis, or 3 orthogonal magnetic dipoles plus 1 electric dipole oriented along $z$-axis, the optimal MUXG cannot be obtained under our scheme. Remind that we need to have at least 2 components for each polarization direction to generate the electric field in an arbitrary direction.

We showed the optimal MUXG can be achievable using only 4 dipole components among 6 . However, as $K$ increases, we cannot remove all the interference links using only 4 components. Observe that each transmitter and receiver is able to eliminate one of its interferences using the fact that the each polarization channel matrix has 2 zero singular values. Therefore, the maximum number of interference links we can remove is $2 K$, but there are $K(K-1)$ cross links in the fully connected $K$-user IC. Hence, we cannot remove all the interference links when $K>3$ using only 4 dipole components. 
If we use all the 6 components of polarimetric antenna at each user, each channel matrix has 4 zero singular values, thus each transmitter and receiver can null out up to 2 interference links in a similar way. Suppose the transmitter $i$ intends to null out the interferences radiated to receivers $j$ and $k$ simultaneously, and let $\mathbf{H}^{[i \rightarrow j k]}=\left[\begin{array}{ll}\mathbf{H}^{[i j]} & \mathbf{H}^{[i k]}\end{array}\right]^{T}$ where $j \neq k$. Since the $\mathbf{H}^{[i \rightarrow j k]}$ is a $12 \times 6$ matrix and its rank is 4 , we can easily find one proper ZF beam-forming matrix for transmitter $i$ whose size is $6 \times 2$ as given by:

$$
\begin{aligned}
\mathbf{V}^{[i \rightarrow j k]}= & \frac{1}{\sqrt{1+\sin ^{2}\left(\phi_{i j}-\phi_{i k}\right)}} \\
& \cdot\left[\begin{array}{cc}
\cos \left(\phi_{i j}\right)-\cos \left(\phi_{i k}\right) & 0 \\
\sin \left(\phi_{i j}\right)-\sin \left(\phi_{i k}\right) & \sin \left(\phi_{i j}-\phi_{i k}\right) \\
0 & \cos \left(\phi_{i j}\right)-\cos \left(\phi_{i k}\right) \\
0 & \sin \left(\phi_{i j}\right)-\sin \left(\phi_{i k}\right) \\
0 & 0
\end{array}\right]
\end{aligned}
$$

In addition, assume receiver $i$ tries to remove the interferences from transmitters $l$ and $m$ simultaneously, where $i, j, k, l$ and $m$ are all different integers. Then, a proper beam-forming matrix for receiver $i$ can be easily obtained in a similar manner, and user $i$ obtains the following parallel channel after applying ZF beam-forming:

$$
\begin{aligned}
\mathbf{\Lambda}^{[i i]} & =\left[\mathbf{U}^{[l m \leftarrow i]}\right]^{*} \mathbf{H}^{[i i]} \mathbf{V}^{[i \rightarrow j k]} \\
= & \frac{a^{[i i]} e^{-j k r_{[i i]}}}{\sqrt{\left(1+\sin ^{2}\left(\phi_{i j}-\phi_{i k}\right)\right)\left(1+\sin ^{2}\left(\phi_{l i}-\phi_{m i}\right)\right)}} \\
& \cdot\left[\begin{array}{cc}
\gamma^{[i i]} & 0 \\
0 & \gamma^{[i i]}
\end{array}\right]
\end{aligned}
$$

where

$$
\begin{aligned}
\gamma^{[i i]}= & \left(\sin \left(\phi_{i i}-\phi_{i j}\right)-\sin \left(\phi_{i i}-\phi_{i k}\right)+\sin \left(\phi_{i j}-\phi_{i k}\right)\right) \\
& \cdot\left(\sin \left(\phi_{i i}-\phi_{l i}\right)-\sin \left(\phi_{i i}-\phi_{m i}\right)+\sin \left(\phi_{l i}-\phi_{m i}\right)\right)
\end{aligned}
$$

The detailed beam-forming scheme is depicted in Fig. 4 If every transmitter and receiver null out 2 interference links, the maximum number of interfering links we can remove is given by $4 K$, therefore this scheme can be valid for the $K \leq 5$ (necessary condition). Fig. 4 shows that the optimal MUXG of $2 K$ can be indeed achieved for $K=5$.

In summary, we have the following two propositions for fixed antenna placement scenario.

Proposition 2: For $K=2$ or 3 with fixed antenna placement, the following antenna configurations at each user achieve the optimal MUXG of $2 K$.

- $\left(e_{x}\right.$ and $\left.e_{y}\right)+\left(m_{y}\right.$ and $\left.m_{y}\right)$

- $\left(e_{x}, e_{y}\right.$, and $\left.e_{z}\right)+\left(m_{x}\right.$ or $\left.m_{y}\right)$

- $\left(e_{x}\right.$ or $\left.e_{y}\right)+\left(m_{x}, m_{y}\right.$, and $\left.m_{z}\right)$

Proposition 3: For $K=4$ or 5 with fixed antenna placement, using all 6 dipole components at each user achieves the optimal MUXG of $2 K$.

In this section, we showed the optimal MUXG can be obtained using only one polarimetric antenna when $K \leq 5$.

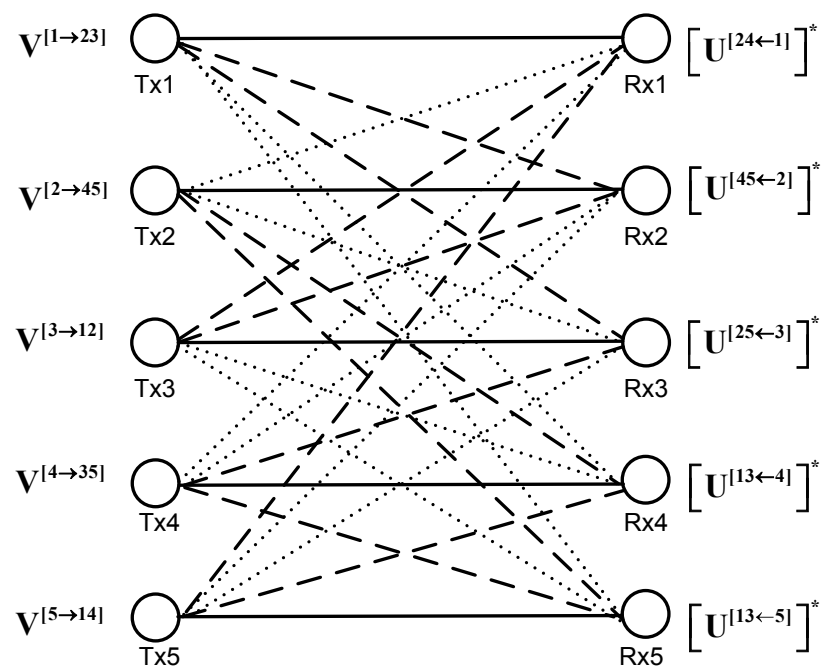

Fig. 4. Fixed antenna placement with single polarimetric antenna for $K=5$. The ZF beam-forming matrices for each transmitter and receiver are depicted. Dashed line $(--)$ denotes the interference links nulled by receivers while dotted line $(\cdots)$ represents the interference links nulled by transmitters.

However, when $K>5$, multiple polarimetric antennas $(M>$ 1) are required.

\section{Fixed Antenna Placement: $M \geq 1$ Case}

In this section, we consider the case where each user uses $M \geq 1$ polarimetric antennas. For simplicity, assume that all 6 components of polarimetric antenna is activated. In this case, the size of each channel matrix becomes $6 M \times 6 M$. However, the rank of this matrix remains at 2 due to the keyhole effect because we only consider the LOS environment and far-field communication. In addition, the geometries of the transmit and receive array also do not affect the rank of the channel matrix. Therefore, we see that each transmitter and receiver can remove up to $3 M-1$ interferences connected to itself because the number of zero singular values is $6 M-2$. In addition, we assume any column vector which lies in the null space of the channel matrix $H^{[i j]}$ is independent of any column vector which spans the null space of $H^{[k l]}$ except when $i=j$ and $k=l$ since the null space of a channel matrix is a function of the angle between transmitter and receiver. Then, the rank of any direct links remains 2 after applying our scheme. Using these properties and assumptions, we have the following proposition.

Proposition 4: For the $K$-user LOS IC with $M$ polarimetric antennas at each user, the optimal MUXG of $2 K$ is achieved if $M=\left\lceil\frac{K+1}{6}\right\rceil$.

\section{Numerical Results}

In this section, we provide some numerical results demonstrating the performance of our scheme. We show achievable multiplicative gains from polarization for 5 -user LOS IC. As can be seen from Fig. 5, the MUXG increases as we use more dipole components and it reaches the maximum of 10 when 


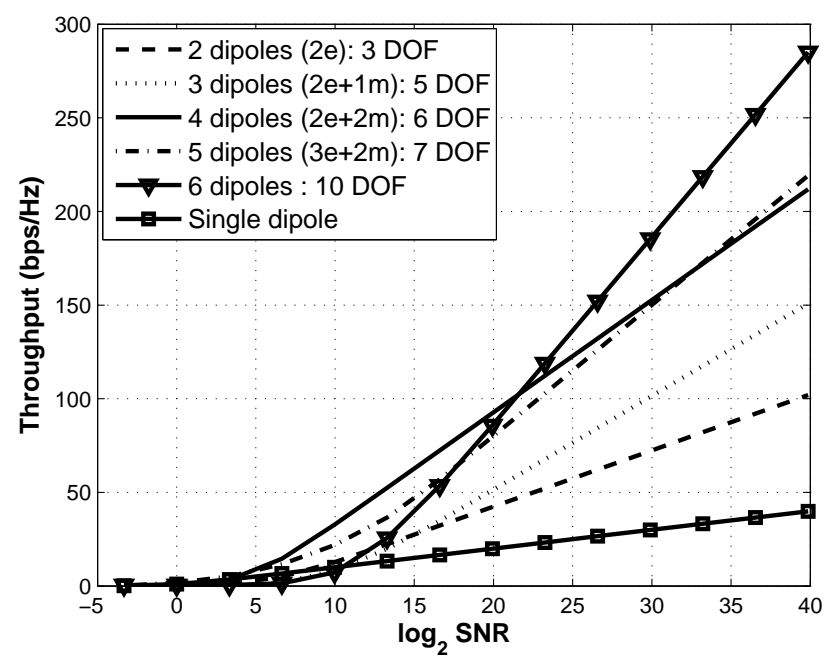

Fig. 5. Multiplicative gains from polarization for 5 user LOS IC.

we use all 6 dipoles. In addition, we can observe that using 2 orthogonal dipoles is not enough.

\section{ACKNOWLEDGMENT}

This research was supported in part by the ITRC (NIPA2009-C1090-0902-0005).

\section{REFERENCES}

[1] L. Zheng and D. N. C. Tse, "Diversity and multiplexing: A fundamental tradeoff in multiple-antenna channels," IEEE Trans. Inf. Theory, vol. 49. no. 5, pp. 1073-1096, May 2003.

[2] S. A. Jafar and M. J. Fakhereddin, "Degrees of freedom for the MIMO interference channel," IEEE Trans. Inf. Theory, vol. 53. no. 7, pp. 26372642, July 2007.

[3] M. Maddah-Ali, A. Motahari, and A. Khandani, "Signaling over MIMO multi-base systems - combination of multi-access and broadcast schemes," in Proc. IEEE Int. Symp. Inform. Theory, 2006.

[4] V. R. Cadambe and S. A. Jafar, "Interference alignment and the degrees of freedom for the $\mathrm{K}$ user interference channel," IEEE Trans. Inf. Theory, vol. IT-54, no. 8, pp. 3425-3441, Aug. 2008.

[5] V. R. Cadambe, S. A. Jafar, and S. Shamai (Shitz), "Interference alignment on the deterministic channel and application to fully connected AWGN interference networks," preprint.

[6] R. Etkin and E. Ordentlich, "On the degrees-of-freedom of the $K$-user Gaussian interference channel," preprint.

[7] L. Grokop, D. N. C. Tse, and R. D. Yates, "Interference alignment for line-of-sight channels," preprint.

[8] M.R. Andrews, P.P. Mitra, and R. deCarvalho, "Tripling the capacity of wireless communication using electromagnetic polarization", Nature, vol 409. pp. 316-318, Jan. 2001.

[9] A. Poon and D. N. C. Tse,"Polarization degrees of freedom", in Proc. IEEE Int. Symp. Inform. Theory, 2008.

[10] Constantine A. Balanis, Antenna Theory: Analysis and Design, 2nd ed. Wiley, 1996.

[11] Roger F. Harrington, Time-Harmonic Electromagnetic fields, Wiley, 2001.

[12] Douglas B. West, Introduction to Graph theory, Prentice Hall. 2001. 